\title{
Preface
}

A supercomputer has been competitively ranked by its computing speed, memory and storage capacity, and parallel processing scalability year by year. Since it was introduced in 1960s, the major players in this field have been mainly dominated by US companies, such as Cray, IBM and Hewlett-Packard, and by Japanese companies, such as NEC, Fujitsu, and Hitachi.

This leadership pattern of supercomputers has been changed since 2004 when Dawning 4000A of China joined the top 10 group in the Top 500 Supercomputer List. This machine was developed by the Institute of Computing Technology, Chinese Academy of Sciences. Since then, besides the United States, China has become the second country that is capable of developing and producing ultra high performance supercomputers. In November 2010, the Tianhe-1A Supercomputer developed by the National University of Defense Technology in China, became the fastest in the world. The other Chinese supercomputer, Dawning Nebulae was ranked the second in the Top 500 List in June 2010.

I am very pleased to introduce three papers representing advanced computing technology of China in this special section. The first paper entitled "The TianHe-1A Supercomputer: Its Hardware and Software" is written by the R\&D team from the National University of Defense Technology. The authors provide technical insights into the design and realization of the TianHe-1A supercomputer, the fastest one in the world by November 2010. The second paper entitled "Dawning Nebulae: A PetaFLOPS Supercomputer with a Heterogeneous Structure" is written by the R\&D team from the Institute of Computing Technology, Chinese Academy of Sciences. The authors describe how they have achieved ultra performance with a heterogeneous structure in a large scale cluster.

In addition to developing advanced supercomputing technology, Chinese researchers have made a 10 years' independent effort to develop CPU chips. This core technology has been mainly owned by major US companies, such as Intel, AMD, and NVIDIA. The third paper entitled "The Godson Processors: Its Research, Development, and Contributions" is written by several architects of the Godson processor chips. They give a roadmap of the Godson project, including its history, advancement of the technology and unique technical merits.

All the authors of the three papers are young researchers, representing the technical competence and strong capability of new generation computer scientists and engineers in China. I would like to thank the JCST editorial management team for the help and professional service to make this special section be published timely.

\section{Guest Editor:}

Xiao-Dong Zhang, Robert M. Critchfield Professor, Department of Computer Science and Engineering The Ohio State University, U.S.A. zhang@cse.ohio-state.edu

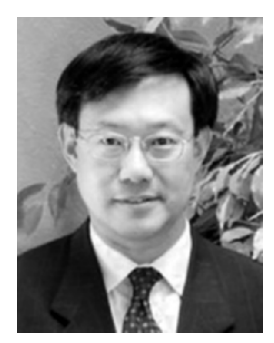

Xiao-Dong Zhang is the Robert M. Critchfield Professor in engineering and Chair of the Computer Science and Engineering Department at the Ohio State University. His research interests focus on data management in computer, networking and distributed systems. He received his Ph.D. degree in computer science from University of Colorado Boulder, where he is a recipient of the Distinguished Engineering Alumni Award in 2011. He is a fellow of the IEEE. 